\title{
THE CHEMODYNAMICAL EVOLUTION OF SPHEROIDAL SYSTEMS AND THE RESULTANT STELLAR ABUNDANCE DISTRIBUTION FUNCTION
}

\author{
T. TSUJIMOTO ${ }^{1}$, T. SHIGEYAMA ${ }^{2}$, AND K. NOMOTO ${ }^{2}$ \\ ${ }^{1}$ National Astronomical Observatory \\ Mitaka, Tokyo 181, Japan \\ ${ }^{2}$ Department of Astronomy, School of Science, \\ University of Tokyo, Bunkyo-ku, Tokyo 113, Japan
}

We construct a chemo-dynamical model for galaxy formation using a three dimensional SPH method. We simulate the formation of two spheroidal systems, i.e., the elliptical galaxy and the Galactic bulge, based on the collapse scenario for protogalaxies. We obtain the chemodynamical formation and evolution models for the two systems during the first $\sim 1$ Gyr. The relative ratio of kinetic to thermal energy of supernovae is found to heavily determine the outcome. By giving the explosion energy of supernovae to the interstellar gas with a physically meaningful relative ratio of kinetic to thermal energy, the elliptical galaxy model has the hot halo and the galactic wind, but it is not the case for the Galactic bulge model.

The chemical and dynamical properties of the model galaxies are found to depend mainly on the gravitational potential set by dark matter (Tsujimoto 1994). For the deeper potential, we obtain a model whose density profiles and velocity dispersions are consistent with the elliptical galaxies. On the other hand, the Galactic bulge-like structure, i.e., a compact core whose mass is $\sim 10^{10} M_{\odot}$ and radius is $\sim 2 \mathrm{kpc}$ can be formed only in a much shallower potential. These results suggest that elliptical galaxies were formed in a deeper potential than spiral galaxies. Our calculations show that these two systems evolve as follows:

a) In the model for the elliptical galaxy, star bursts occur in the central part of dark matter. Eventually a core which consists of stars and the hot halo are formed. During the formation, the galactic wind with heavy elements (nearly solar abundance) occurs and $\sim 10 \%$ of the initial gas escapes from the galactic potential. 


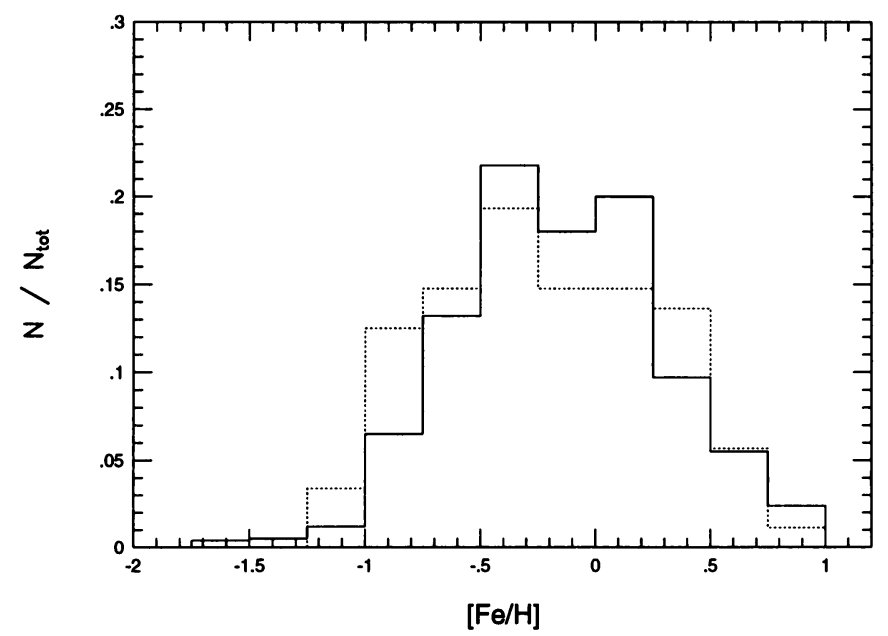

Figure 1. Calculated abundance distribution function of stars in the core for the Galactic bulge model (solid line) as compared with that of the bulge K-giants (dotted line:McWilliam \& Rich 1994).

b) In the model for the Galactic bulge, (i) gases collapse in almost uniformly distributed dark matter, (ii) a small fraction of gases forms stars in the central part, and (iii) the residual gases make a bounce due to gas pressure, centrifugal force and the kinetic energy of supernova explosions.

Our model for the Galactic bulge is successful in reproducing the revised abundance distribution function of the bulge K-giants (McWilliam \& Rich 1994). We find that the resultant stellar abundance distribution is produced by the inhomogenous enrichment. The wide range of metallicity and super metal-rich stars are made by the effect. The density inhomogenity in the core results in the inhomogenous metal enrichment. Such an inhomogeneity does not appear in one zone models. In addition, it is found that the distribution with its peak lying at a low metallicity $([\mathrm{Fe} / \mathrm{H}] \sim-0.5)$, despite the existence of super metal-rich stars, can be reproduced under the condition that the gas contaminated by heavy elements can easily escape from the core. If the bulge evolves in the closed system, the metallicity at the peak is shifted to higher and the abundance distribution function becomes similar to the observed one by Rich (1988). Not only the size and the mass of the Galactic bulge but also the abundance distribution function of the bulge $\mathrm{K}$-giant implies that the bulge is formed in the shallow galactic potential.

\section{References}

McWilliam, A. \& Rich, R.M., 1994, ApJS 91, 749

Rich, R.M., 1988, AJ 362, 606

Tsujimoto, T., 1994, submitted to ApJ 